\title{
Bayesian Models for Structured Sparse Estimation via Set Cover Prior
}

\author{
Xianghang Liu ${ }^{1,2}$, Xinhua Zhang ${ }^{1,3}$, and Tibério Caetano ${ }^{1,2,3}$ \\ 1 Machine Learning Research Group, National ICT Australia, Sydney and Canberra, Australia \\ 2 School of Computer Science and Engineering, The University of New South Wales, Australia \\ 3 Research School of Computer Science, The Australian National University, Australia \\ \{xianghang.liu, xinhua.zhang, tiberio.caetano\}@nicta.com.au
}

\begin{abstract}
A number of priors have been recently developed for Bayesian estimation of sparse models. In many applications the variables are simultaneously relevant or irrelevant in groups, and appropriately modeling this correlation is important for improved sample efficiency. Although group sparse priors are also available, most of them are either limited to disjoint groups, or do not infer sparsity at group level, or fail to induce appropriate patterns of support in the posterior. In this paper we tackle this problem by proposing a new framework of prior for overlapped group sparsity. It follows a hierarchical generation from group to variable, allowing group-driven shrinkage and relevance inference. It is also connected with set cover complexity in its maximum a posterior. Analysis on shrinkage profile and conditional dependency unravels favorable statistical behavior compared with existing priors. Experimental results also demonstrate its superior performance in sparse recovery and compressive sensing.
\end{abstract}

\section{Introduction}

Sparsity is an important concept in high-dimensional statistics [1] and signal processing [2] that has led to important application successes. It reduces model complexity and improves interpretability of the result, which is critical when the number of explanatory variables $p$ in the problem is much higher than the number of training instances $n$.

From a Bayesian perspective, the sparsity of a variable $\beta_{i}$ is generally achieved by shrinkage priors, which often take the form of scale mixture of Gaussians: $\beta_{i} \mid z_{i} \sim$ $\mathcal{N}\left(0, z_{i}\right) . z_{i}$ indicates the relevance of $\beta_{i}$, and a broad range of priors on $z_{i}$ has been proposed. For example, the spike and slab prior [3,4] uses a Bernoulli variable for $z_{i}$, which allows $\beta_{i}$ to be exactly zero with a positive probability. Absolutely continuous alternatives also abound [5], e.g., the horseshoe prior [6, 7] which uses half-Cauchy on $z_{i}$ and offers robust shrinkage in the posterior. Interestingly, the maximum a posterior (MAP) inference often corresponds to deterministic models based on sparsity-inducing regularizers, e.g. Lasso [8] when $z_{i}$ has a gamma distribution $[9,10]$. In general, the log-posterior can be non-concave [11, 12].

However, many applications often exhibit additional structures (correlations) in variables rather than being independent. Groups may exist such that variables of each group are known a priori to be jointly relevant or irrelevant for data generation. Encoding this

T. Calders et al. (Eds.): ECML PKDD 2014, Part II, LNCS 8725, pp. 273-289, 2014.

(C) Springer-Verlag Berlin Heidelberg 2014 
knowledge in the prior proves crucial for improved accuracy of estimation. The simplest case is when the groups are disjoint, and they form a partition of the variable set. This allows the relevance indicator $z_{i}$ of all variables in each group to be tied, forming a group indicator which is endowed with a zero-centered prior as above [13, 14]. In particular, a gamma prior now yields the Bayesian group Lasso [15], and its MAP is the group Lasso [16] which allows group information to provably improve sample efficiency [17]. More refined modeling on the sparsity within each group has also been explored $[18,19]$. We overview the related background in Section 2.

However, groups do overlap in many practical applications, e.g. gene regulatory network in gene expression data [20], and spatial consistency in images [21]. Techniques that deal with this scenario start to diverge. A commonly used class of method employs a Markov random field (MRF) to enforce smoothness over the relevance indicator of all variables within each group [22-24]. However, this approach does not infer relevance at the group level, and does not induce group-driven shrinkage.

Another popular method is to directly use the Bayesian group Lasso, despite the loss of hierarchical generative interpretation due to the overlap. Its MAP inference has also led to a rich variety of regularizers that promote structured sparsity [21, 25], although statistical justification for the benefit of using groups is no longer rich and solid. Moreover, Bayesian group Lasso tends to shrink a whole group based on a complexity score computed from its constituent variables. So the support of the posterior $\boldsymbol{\beta}$ tends to be the complement of the union of groups, rather than the union of groups as preferred by many applications.

To address these issues, we propose in Section 3 a hierarchical model by placing relevance priors on groups only, while the variable relevance is derived (probabilistically) from the set of groups that involve it. This allows direct inference of group relevance, and is amenable to the further incorporation of hierarchies among groups. All previously studied sparsity-inducing priors on relevance variables can also be adopted naturally, leading to a rich family of structured sparse prior. The MAP of our model turns out exactly the set cover complexity, which provably reduces sample complexity for overlapped groups [26].

Although in appearance our model simply reverses the implication of relevance in Bayesian group Lasso, it amounts to considerably more desirable shrinkage profile [7]. In Section 4, detailed analysis based on horseshoe prior reveals that set cover priors retain the horseshoe property in its posterior, shrinking reasonably for small response and diminishing when response grows. Surprisingly, these properties are not preserved by the other structured alternatives. Also observed in set cover prior is the favorable conditional dependency between relevance variables, which allows them to "explainaway" each other through the overlap of two groups they each belong to. Experimental results in Section 5 confirm that compared with state-of-the-art structured priors, the proposed set cover prior outperforms in sparse recovery and compressive sensing on both synthetic data and real image processing datasets.

Note different from [27] and [28], we do not introduce regression variables that account for interactions between features, i.e. $\beta_{i j}$ for $x_{i} x_{j}$. 


\section{Preliminaries on Sparse Priors}

In a typical setting of machine learning, we are given $n$ training examples $\left\{\mathbf{x}_{i}, y_{i}\right\}_{i=1}^{n}$, where $\mathbf{x}_{i} \in \mathbb{R}^{p}$ represents a vector of $p$ features/variables, and $y_{i}$ is the response that takes value in $\mathbb{R}$ for regression, and in $\{-1,1\}$ for classification. Our goal is to learn a linear model $\boldsymbol{\beta} \in \mathbb{R}^{p}$, or a distribution of $\boldsymbol{\beta}$, such that $\mathbf{x}_{i}^{\prime} \boldsymbol{\beta}$ agrees with $y_{i}$. This problem is usually ill-posed, especially when $p \gg n$ as considered in this work. Therefore prior assumptions are required and here we consider a popular prior that presumes $\boldsymbol{\beta}$ is sparse. In Bayesian methods, the compatibility between $y_{i}$ and $\mathbf{x}_{i}^{\prime} \boldsymbol{\beta}$ is enforced by a likelihood function, which is typically normal for regression (i.e., $y \mid \mathbf{x}, \boldsymbol{\beta} \sim \mathcal{N}\left(\mathbf{x}^{\prime} \boldsymbol{\beta}, \sigma^{2}\right)$ ), and Bernoulli for classification. $\sigma$ is a pre-specified constant.

The simplest form of sparsity is enforced on each element of $\boldsymbol{\beta}$ independently through priors on $\beta_{i}$. Most existing models use a scalar mixture of normals that correspond to the graphical model $z_{i} \rightarrow \beta_{i}[27,29,30]:$

$$
\pi\left(\beta_{i}\right)=\int \mathcal{N}\left(\beta_{i} ; 0, \sigma_{0}^{2} z_{i}\right) f\left(z_{i}\right) \mathrm{d} z_{i}
$$

Here $\sigma_{0}^{2}$ can be a constant, or endowed with a prior. Key to the model is the latent conditional variance $z_{i}$, which is often interpreted as relevance of the variable $\beta_{i}$. Larger $z_{i}$ allows $\beta_{i}$ to take larger absolute value, and by varying the mixing distribution $f$ of $z_{i}$ we obtain a range of priors on $\boldsymbol{\beta}$, differing in shrinkage profile and tail behavior. For example, the spike and slab prior $[3,4]$ adopts

$$
f_{\mathrm{SS}}\left(z_{i}\right)=p_{0} \delta\left(z_{i}-1\right)+\left(1-p_{0}\right) \delta\left(z_{i}\right)
$$

where $\delta$ is the Dirac impulse function and $p_{0}$ is the prior probability that $\beta_{i}$ is included. Absolutely continuous distributions of $z_{i}$ are also commonly used. An inverse gamma distribution on $z_{i}$ leads to the Student- $t$ prior, and automatic relevance determination [ARD, 9] employs $f\left(z_{i}\right) \propto z_{i}^{-1}$. Indeed, a number of sparsity-inducing priors can be unified using the generalized beta mixture $[5,31]$ :

$$
z_{i} \mid \lambda_{i} \sim \mathrm{Ga}\left(a, \lambda_{i}\right), \quad \text { and } \quad \lambda_{i} \sim \mathrm{Ga}(b, d) .
$$

Here Ga stands for the gamma distribution with shape and rate (inverse scale) parameters. In fact, $z_{i}$ follows the generalized beta distribution of the second kind:

$$
\operatorname{GB} 2\left(z_{i} ; 1, d, a, b\right)=z_{i}^{a-1}\left(1+z_{i} / d\right)^{-a-b} d^{-a} / B(a, b),
$$

where $B(a, b)$ is the beta function. When $a=b=\frac{1}{2}$, it yields the horseshoe prior on $\boldsymbol{\beta}$ [6]. The normal-exponential-gamma prior and normal-gamma prior [32] can be recovered by setting $a=1$ and $b=d \rightarrow \infty$ respectively. In the intersection of these two settings is the Bayesian Lasso: $\pi(\boldsymbol{\beta}) \sim \exp \left(-\|\boldsymbol{\beta}\|_{1}\right)[10]$, where $\|\boldsymbol{\beta}\|_{p}:=\left(\sum_{i}\left|\beta_{i}\right|^{p}\right)^{\frac{1}{p}}$ for $p \geq 1$.

To lighten notation, in the case of spike and slab we will also use $z_{i}$ to represent Bernoulli variables valued in $\{0,1\}$. So integrating over $z_{i} \geq 0$ with respect to the density in (2) can be interpreted as weighted sum over $z_{i} \in\{0,1\}$. 


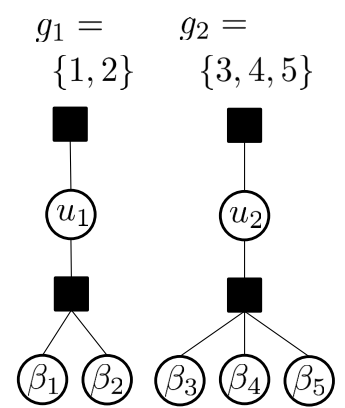

Fig. 1. Group spike and slab

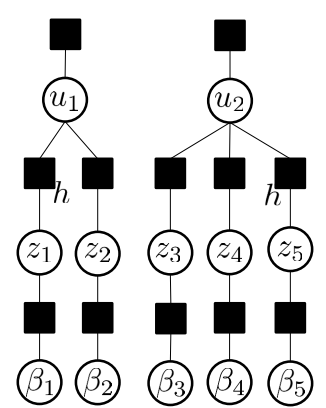

Fig. 2. Nested spike and slab

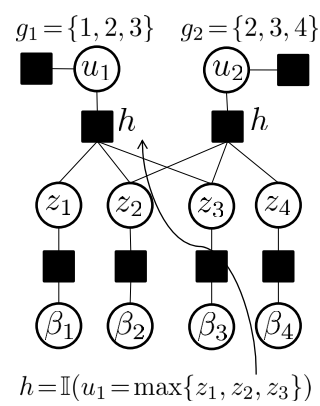

Fig. 3. Group counting prior for spike and slab

\subsection{Disjoint Groups}

In many applications, prior knowledge is available that the variables can be partitioned into disjoint groups $g_{i} \subseteq[p]:=\{1, \ldots, p\}$, and all variables in a group tend to be positively correlated, i.e. relevant or irrelevant simultaneously. Denote $\mathcal{G}=\left\{g_{1}, g_{2}, \ldots\right\}$. [13] generalized the spike and slab prior to this scenario by introducing a scalar parameter of relevance for each group: $u_{g} \sim f_{\mathrm{SS}}$, and extending (1) into a scalar mixture of multivariate normal

$$
\pi\left(\boldsymbol{\beta}_{g}\right)=\int \mathcal{N}\left(\boldsymbol{\beta}_{g} ; \mathbf{0}, \boldsymbol{\Lambda}_{g} u_{g}\right) f\left(u_{g}\right) \mathrm{d} u_{g} \forall g \in \mathcal{G} .
$$

Here $\boldsymbol{\beta}_{g}$ encompasses all variables in the group $g$, and $\boldsymbol{\Lambda}_{g}$ is a diagonal matrix of variance. See Figure 1 for the factor graph representation that will facilitate a unified treatment of other models below. As a result, correlation is introduced among all variables in each group. Using exactly the same density $f$ as above (but on $u_{g}$ here), one may recover the group horseshoe, group ARD [14], and Bayesian group Lasso [15]:

$$
\pi(\boldsymbol{\beta}) \propto \exp \left(-\|\boldsymbol{\beta}\|_{\mathcal{G}}\right), \text { where }\|\boldsymbol{\beta}\|_{\mathcal{G}}=\sum_{g}\left\|\boldsymbol{\beta}_{g}\right\|_{p} .
$$

Common choices of $p$ are 2 and $\infty$. To further model the sparsity of different variables within a group, [18] proposed a nested spike and slab model as shown in Figure 2. The key idea is to employ both Bernoulli variables $z_{i}$ and $u_{g}$ that encode the relevance of variables and groups respectively, and to define the spike and slab distribution of $\beta_{i}$ conditional on $u_{g}=1$. In particular, $z_{i}$ must be 0 if $u_{g}=0$, i.e. group $g$ is excluded. This relation is encoded by a factor between $z_{i}$ and $u_{g}$ :

$$
h\left(z_{i}, u_{g}\right)=\left\{\begin{array}{ll}
p_{0}^{z_{i}}\left(1-p_{0}\right)^{1-z_{i}} & \text { if } u_{g}=1 \\
\mathbb{I}\left(z_{i}=0\right) & \text { if } u_{g}=0
\end{array}, \forall i \in g .\right.
$$

Here $\mathbb{I}(\cdot)=1$ if $\cdot$ is true, and 0 otherwise. 


\section{Structured Prior with Overlapped Groups}

In many applications, groups may overlap and fully Bayesian treatments for this setting have become diverse.

Group Counting Prior (GCP). A straightforward approach is to ignore the fact of overlapping, and simply use the group Lasso prior in (6). This idea is also used in deterministic overlapped group Lasso [16]. When $p=\infty$, the norm in (6) is the Lovász extension of the group counting penalty [33] which, in the case of spike and slab prior on $\beta_{i}$, can be written in terms of the binary relevance indicator $\mathbf{z}:=\left\{z_{i}\right\} \in\{0,1\}^{p}$

$$
\Omega(\mathbf{z})=\prod_{g \in \mathcal{G}} p_{0}^{u_{g}}\left(1-p_{0}\right)^{1-u_{g}}, \text { where } u_{g}=\max _{i: i \in g} z_{i} .
$$

So a group is deemed as relevant $\left(u_{g}=1\right)$ if, and only if, any variable in the group is relevant $\left(z_{i}=1\right)$. The factor graph is given in Figure 3, with a Bernoulli potential on $u_{g}$. However, since this prior promotes $u_{g}$ to be 0 (i.e. zero out all variables in the group $g$ ), the support of $\boldsymbol{\beta}$ in the posterior tends to be the complement of a union of groups. Although this may be appropriate for some applications, the support is often more likely to be the union of groups.

MRF Prior. Instead of excluding groups based on its norm, the MRF prior still places sparsity-inducing priors on each variable $\beta_{i}$, but further enforces consistency of relevance within each group via $z_{i}$. For example, assuming the variables are connected via an undirected graph where each edge $(i, j) \in E$ constitutes a group, [22, 34] extended the spike and slab prior by incorporating a pairwise MRF over the relevance indicators $z_{i}: \exp \left(-\sum_{(i, j) \in E} R_{i j} \mathbb{I}\left(z_{i} \neq z_{j}\right)\right)$.

As a key drawback of the above two priors, they do not admit a generative hierarchy and perform no inference at the group level. To address these issues, we next construct a hierarchical generative model which explicitly characterizes the relevance of both groups and variables, as well as their conditional correlations.

\subsection{Set Cover Prior (SCP)}

To better clarify the idea, we first focus on spike and slab prior where sparsity can be easily modeled by Bernoulli variables $z_{i}$ and $u_{g}$. Recall the nested model in Figure 2, where each group has a Bernoulli prior, and each variable $z_{i}$ depends on the unique group that it belongs to. Now since multiple groups may be associated with each node, it will be natural to change the dependency into some arithmetics of these group indicators. In Figure 4, we show an example with ${ }^{1}$

$$
h\left(z_{i},\left\{u_{g}: i \in g\right\}\right)=\mathbb{I}\left(z_{i} \leq \max \left\{u_{g}: i \in g\right\}\right) .
$$

This means a variable can be relevant only if any group including it is also relevant. Although this appears simply reversing the implication relations between group and variable in the group counting prior, it does lead to a hierarchical model and enjoys much more desirable statistical properties as will be shown in Section 4.

\footnotetext{
${ }^{1}$ This defines a potential in an MRF; there is no explicit prior on $z_{i}$.
} 


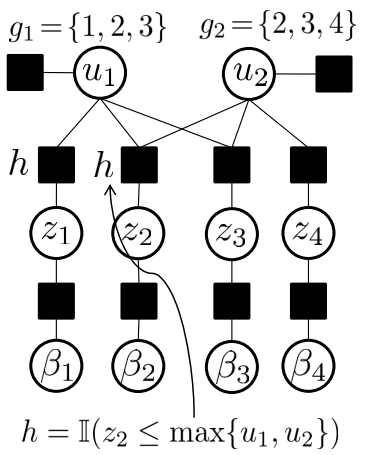

Fig. 4. Set cover prior for spike and slab

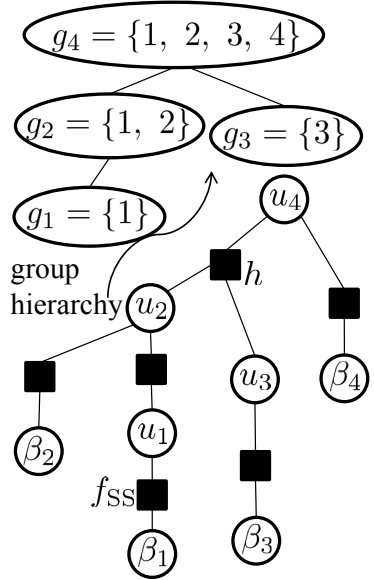

Fig. 5. Set cover prior for spike and slab with tree hierarchy. $u_{j}$ corresponds to $g_{j} . h=\mathbb{I}\left(u_{4} \geq\right.$ $\left.\max \left\{u_{2}, u_{3}\right\}\right)$.

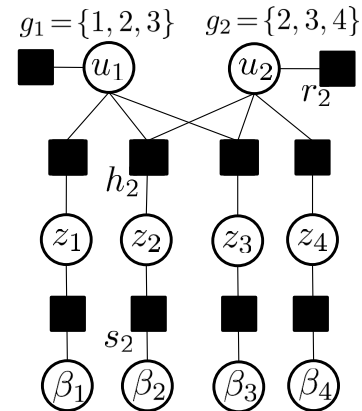

Fig. 6. Set cover prior using horseshoe. $r_{2}=\mathrm{Ga}\left(u_{2} ; \frac{1}{2}, \frac{1}{2}\right) . h_{2}=$ $\operatorname{Ga}\left(z_{2} ; \frac{1}{2}, \max \left\{u_{1}, u_{2}\right\}\right)$.

By endowing a Bernoulli prior on all $u_{g}$ with $\operatorname{Pr}\left(u_{g}=1\right)=p_{0}<0.5$ (i.e. favoring sparsity), we complete a generative prior of $\boldsymbol{\beta}$ in a spike and slab fashion. Given an assignment of $\mathbf{z}$, it is interesting to study the mode of $\left\{u_{g}\right\}$, which is the solution to

$$
\min _{\left\{u_{g}\right\}} \sum_{g} u_{g}, \quad \text { s.t. } u_{g} \in\{0,1\}, \sum_{g: i \in g} u_{g} \geq z_{i}, \forall i .
$$

This turns out to have exactly the same complexity as set cover [26]. It seeks the smallest number of groups such that their union covers the set of variables. Hence we will call this prior as "set cover prior". This optimization problem is NP-hard in general, and some benefit in sample complexity is established by [26].

A number of extensions follow directly. Additional priors (e.g. MRF) can be placed on variables $z_{i}$. The max in (9) can be replaced by min, meaning that a variable can be selected only if all groups involving it are selected. Other restrictions such as limiting the number of selected variables in each (selected) group can also be easily incorporated [35]. Moreover, groups can assume a hierarchical structure such as tree, i.e. $g \cap g^{\prime} \in$ $\left\{g, g^{\prime}, \emptyset\right\}$ for all $g$ and $g^{\prime}$. Here the assumption is that if a node $g^{\prime}$ is included, then all its ancestors $g \supset g^{\prime}$ must be included as well $[21,36]$. This can be effectively enforced by adding a factor $h$ that involves each group $g$ and its children $\operatorname{ch}(g)$ (see Figure 5):

$$
h(g, \operatorname{ch}(g))=\mathbb{I}\left(u_{g} \geq \max _{g^{\prime} \in \operatorname{ch}(g)} u_{g^{\prime}}\right) .
$$

When the groups are disjoint, both set cover and group counting priors are equivalent to group spike and slab.

\subsection{Extension to Generalized Beta Mixture}

The whole framework is readily extensible to the continuous sparse priors such as horseshoe and ARD. Using the interpretation of $z_{i}$ and $u_{g}$ as relevance measures, we could 
simply replace the function $\mathbb{I}$ that tests equality by the Dirac impulse function $\delta$, and apply various types of continuous valued priors on $z_{i}$ and $u_{g}$. This is indeed feasible for GCP, e.g. encode the continuous variant of (8) using the generalized beta mixture in (3)

$$
h\left(u_{g},\left\{z_{i}: i \in g\right\}\right)=\delta\left(u_{g}-\max \left\{z_{i}: i \in g\right\}\right), \quad h\left(u_{g}\right)=\operatorname{GB} 2\left(u_{g} ; 1, d, a, b\right) .
$$

Here more flexibility is available when $z_{i}$ is continuous valued, because the max can be replaced by multiplication or summation, which promotes or suppresses sparsity respectively [27, Theorem 1,2].

However problems arise in SCP if we directly use

$$
z_{i}=\max _{g: i \in g} u_{g} \text { or } \min _{g: i \in g} u_{g}, \quad \text { where } u_{g} \sim \operatorname{GB} 2\left(u_{g} ; 1, d, a, b\right),
$$

because it leads to singularities in the prior distribution on z. To smooth the prior, we resort to arithmetic combinations of the intermediate variables in the generative process of the prior on $u_{g}$. Note that in (3), $d$ is a scale parameter, while $a$ and $b$ control the behavior of the distribution of $z_{i}$ close to zero and on the tail, respectively. A smaller value of $\lambda_{i}$ places more probability around 0 in $z_{i}$, encouraging a sparser $\beta_{i}$. So a natural way to combine the group prior is:

$$
z_{i} \mid\left\{u_{g}\right\} \sim \operatorname{Ga}\left(a, \max _{g: i \in g} u_{g}\right), \text { and } u_{g} \sim \mathrm{Ga}(b, d),
$$

where max allows $z_{i}$ to pick up the most sparse tendency encoded in all $u_{g}$ of the associated groups ${ }^{2}$. Changing it to min leads to adopting the least sparse one. The resulting graphical model is given in Figure 6. Here $u_{g}$ has a gamma distribution, playing the same role of relevance measure as in the normal-gamma prior on $\beta_{i}$ [32]. The SCP constructed in (14) is no longer equivalent to the group priors in Section 2.1, even when the groups are disjoint.

In fact, the arithmetics that combine multiple groups can be carried out at an even higher level of the generative hierarchy. For example, in the horseshoe prior where $a=b=1 / 2$, one may introduce an additional layer of mixing over the scale parameter $d$, making it an arithmetic combination of $u_{g}$ of the associated groups. We leave this possibility for future exploration.

Notice [38] used a partial least squares approach based on an MRF of binary selectors of groups and variables. However their method is confined to spike and slab, because these two groups of indicators are not coupled by the potential function, but by imposing external restrictions on the admissible joint assignment that is valued in $\{0,1\}$. It also brings much challenge in MCMC inference.

\section{Analysis of Structured Sparse Prior}

Although the above three types of priors for structured sparsity appear plausible, their statistical properties differ significantly as we study in this section. Here in addition to the robust shrinkage profile studied by [6], we also compare the conditional correlation among variables when the groups overlap.

\footnotetext{
${ }^{2}$ See more detailed discussions in Appendix A of the full paper [37] on how a greater value of the second argument (rate, i.e. inverse scale) of a Gamma distribution induces higher sparsity in $\beta_{i}$.
} 


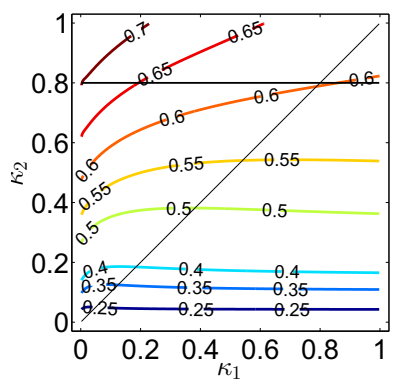

(a) Contour of $\mathbb{E}\left[\kappa_{3} \mid \kappa_{1}, \kappa_{2}\right]$

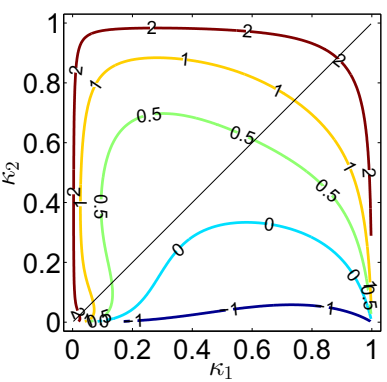

(b) Contour of $\log p\left(\kappa_{1}, \kappa_{2}\right)$

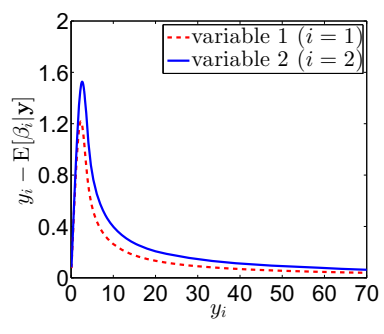

(c) $y_{i}-\mathbb{E}\left[\beta_{i} \mid \mathbf{y}\right]$ v.s. $y_{i}$

Fig. 7. Set cover prior. The contour levels in panel (b) are: $-1,0,0.5,1,2$.

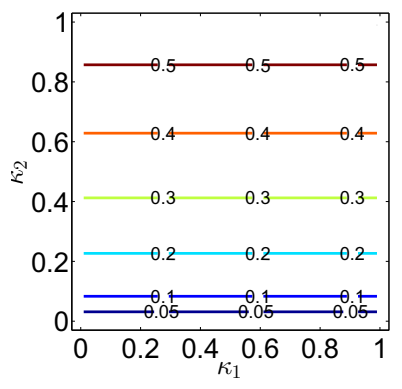

(a) Contour of $\mathbb{E}\left[\kappa_{3} \mid \kappa_{1}, \kappa_{2}\right]$

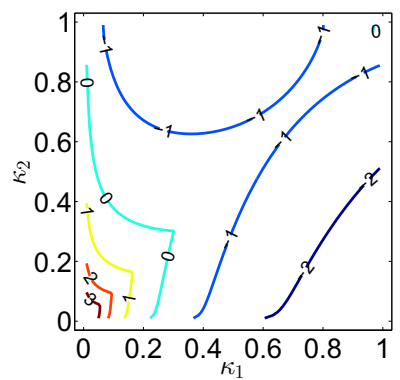

(b) Contour of $\log p\left(\kappa_{1}, \kappa_{2}\right)$

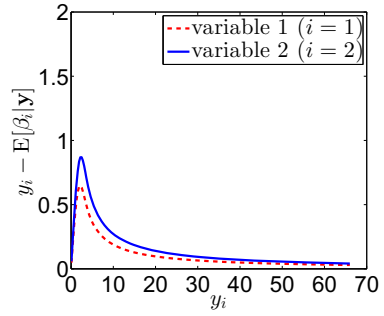

(c) $y_{i}-\mathbb{E}\left[\beta_{i} \mid \mathbf{y}\right]$ v.s. $y_{i}$

Fig. 8. Group counting prior. The contour levels in panel (b) are: $-2,-1,0,1,2,3$.

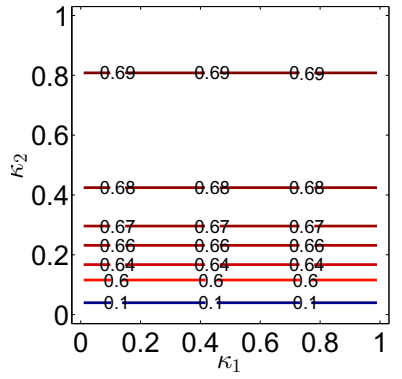

(a) Contour of $\mathbb{E}\left[\kappa_{3} \mid \kappa_{1}, \kappa_{2}\right]$

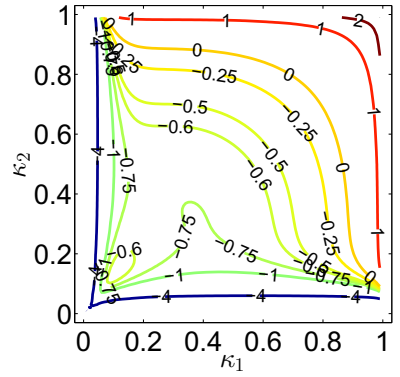

(b) Contour of $\log p\left(\kappa_{1}, \kappa_{2}\right)$

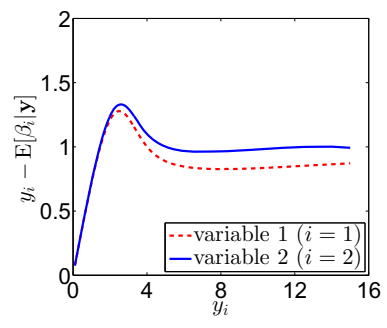

(c) $y_{i}-\mathbb{E}\left[\beta_{i} \mid \mathbf{y}\right]$ v.s. $y_{i}$

Fig. 9. MRF prior $(\alpha=0.01)$. The contour levels in panel (b) are $-4,-1,-0.75, \ldots, 0,1,2$.

Consider $p=3$ variables, and there are two groups $\{1,2\}$ and $\{2,3\}$ which overlap on variable 2 . The design matrix $X$ is the $3 \times 3$ identity matrix $I(n=3)$, and the observation $\mathbf{y} \mid \boldsymbol{\beta} \sim \mathcal{N}(X \boldsymbol{\beta}, I)=\mathcal{N}(\boldsymbol{\beta}, I)$. Let $\sigma_{0}=1$. Then the expected posterior value of $\boldsymbol{\beta}$ given $\mathbf{z}$ has a closed form $\mathbb{E}\left[\beta_{i} \mid z_{i}, y_{i}\right]=\left(1-\kappa_{i}\right) y_{i}$ where $\kappa_{i}=1 /\left(1+z_{i}\right)$ is a 
random shrinkage coefficient. The distribution of $\kappa_{i}$ is determined entirely by the prior on $z_{i}$, and a larger value of $\kappa_{i}$ means a greater amount of shrinkage towards the origin.

As a concrete example, we study the horseshoe prior with $a=b=d=1 / 2$. GCP and SCP use the formulae (12) and (14), respectively. The MRF prior attaches a horseshoe potential on each $\beta_{i}$, and in addition employs a smooth MRF $\exp \left(-\alpha\left(z_{1}-\right.\right.$ $\left.\left.z_{2}\right)^{2}-\alpha\left(z_{2}-z_{3}\right)^{2}\right)$ with $\alpha=0.01$. We use a Gaussian MRF because there is no need of shrinking the difference.

\subsection{Conditional Dependency (Explain-Away Effect)}

We first consider the conditional distribution of $\kappa_{3}$ given $\kappa_{1}$ and $\kappa_{2}$. Since it is hard to visualize a function of three variables, we show in panel (a) of Figures 7 to 9 the mean $\mathbb{E}\left[\kappa_{3} \mid \kappa_{2}, \kappa_{1}\right]$ under the three priors. Clearly the mean of $\kappa_{3}$ does not change with $\kappa_{1}$ in GCP and MRF prior, because $z_{3}$ is simply independent of $z_{1}$ given $z_{2}$. The mean of $\kappa_{3}$ grows monotonically with $\kappa_{2}$, as MRF favors small difference between $z_{2}$ and $z_{3}$ (hence between $\kappa_{2}$ and $\kappa_{3}$ ), and in SCP smaller $\kappa_{2}$ clamps a larger value of $\max \left\{z_{2}, z_{3}\right\}$, shifting more probability mass towards greater $z_{3}$ which results in a lower mean of $\kappa_{3}$.

Interestingly when $\kappa_{2}$ is large, the SCP allows the mean of $\kappa_{3}$ to decrease when $\kappa_{1}$ grows. See, e.g., the horizonal line at $\kappa_{2}=0.8$ in Figure 7a. To interpret this "explainaway" effect, first note a greater value of $\kappa_{2}$ means $z_{2}$ has a higher inverse scale. Due to the max in (14), it implies that either $u_{1}$ or $u_{2}$ is large, which means $\kappa_{1}$ or $\kappa_{3}$ is large since variables 1 and 3 belong to a single group only. Thus when $\kappa_{1}$ is small, $\kappa_{3}$ receives more incentive to be large, while this pressure is mitigated when $\kappa_{1}$ itself increases. On the other hand when $\kappa_{2}$ is small, $\kappa_{1}$ and $\kappa_{3}$ must be both small, leading to the flat contour lines.

\subsection{Joint Shrinkage}

Next we study the joint density of $\kappa_{1}$ and $\kappa_{2}$ plotted in panel (b). SCP exhibits a 2-D horseshoe shaped joint density in Figure 7b, which is desirable as it prefers either large shrinkage or little shrinkage. In GCP, however, the joint density of $\left(\kappa_{1}, \kappa_{2}\right)$ concentrates around the origin in Figure 8b. Indeed, this issue arises even when there are only two variables, making a single group. Fixing $\kappa_{2}$ and hence $z_{2}$, $\max \left\{z_{1}, z_{2}\right\}$ does not approach 0 even when $z_{1}$ approaches 0 (i.e. $\kappa_{1}$ approaches 1 ). So it is unable to exploit the sparsity-inducing property of horseshoe prior which places a sharply growing density towards the origin. The joint density of MRF is low when $\kappa_{1}$ and $\kappa_{2}$ are both around the origin, although the marginal density of each of them seems still high around zero.

\subsection{Robust Marginal Shrinkage}

Finally we investigate the shrinkage profile via the posterior mean $\mathbb{E}[\boldsymbol{\beta} \mid \mathbf{y}]$, with $\mathbf{z}$ integrated out. Let $q(\mathbf{z})$ be proportional to the prior density on $\mathbf{z}$ (note the group counting 
and MRF priors need a normalizer). Then $\mathbb{E}\left[\beta_{i} \mid \mathbf{y}\right]=\gamma_{i}^{(1)} / \gamma_{i}^{(0)}$, where for $k \in\{0,1\}$

$$
\begin{gathered}
\gamma_{i}^{(k)}=\int \beta_{i}^{k} q(\mathbf{z}) \prod_{j} \mathcal{N}\left(\beta_{j} ; 0, z_{j}\right) \mathcal{N}\left(y_{j} ; \beta_{j}, 1\right) \mathrm{d} \beta_{j} \mathrm{~d} \mathbf{z}, \\
\text { and } \int \beta_{j}^{k} \mathcal{N}\left(\beta_{j} ; 0, z_{j}\right) \mathcal{N}\left(y_{j} ; \beta_{j}, 1\right) \mathrm{d} \beta_{j}=\sqrt{\frac{1}{8 \pi}} \frac{z_{j}^{k}}{\left(1+z_{j}\right)^{k+\frac{1}{2}}} \exp \left(\frac{-y_{j}^{2}}{2+2 z_{j}}\right) .
\end{gathered}
$$

Panel (c) of Figures 7 to 9 shows $y_{i}-\mathbb{E}\left[\beta_{i} \mid y_{i}\right]$ (the amount of shrinkage) as a function of $y_{i}$, for variables $i \in\{1,2\}$. All $y_{j}(j \neq i)$ are fixed to 1 . In Figure 7c, Both SCP and GCP provide valuable robust shrinkage, with reasonable shrinkage when $y_{i}$ is small in magnitude, and diminishes as $y_{i}$ grows. And as expected, variable 2 shrinks more than variable 1. In SCP, variable 2 takes the sparser state between variables 1 and 3 via the max in (14), while in GCP variable 2 contributes to both sparsity-inducing priors of $u_{1}$ and $u_{2}$ in (12). Notice that for small $y_{1}$, GCP is not able to yield as much shrinkage as SCP. This is because for small $y_{1}, z_{1}$ is believed to be small, and hence the value of $\max \left\{z_{1}, z_{2}\right\}$ is dominated by the belief of $z_{2}$ (which is larger). This prevents $z_{1}$ from utilizing the horseshoe prior around zero. The case for $y_{2}$ is similar.

In fact, we can theoretically establish the robust shrinkage of SCP for any group structure under the current likelihood $\mathbf{y} \mid \boldsymbol{\beta} \sim \mathcal{N}(\boldsymbol{\beta}, I)$.

Theorem 1. Suppose SCP uses horseshoe prior in (14) with $a=b=1 / 2$. Then for any group structure, $\lim _{y_{i} \rightarrow \infty}\left(y_{i}-\mathbb{E}\left[\beta_{i} \mid \mathbf{y}\right]\right)=0$ with fixed values of $\left\{y_{j}: j \neq i\right\}$.

Proof. (sketch) The key observation based on (15) and (16) is that $\mathbb{E}\left[\beta_{i} \mid \mathbf{y}\right]-y_{i}=$ $\frac{\partial}{\partial y_{i}} \log F(\mathbf{y})$ where $F(\mathbf{y})$ is given by

$$
\begin{aligned}
& \int_{\mathbf{z}} \prod_{j}\left(1+z_{j}\right)^{\frac{-1}{2}} \exp \left(\frac{-y_{j}^{2}}{2+2 z_{j}}\right) q(\mathbf{z}) \mathrm{d} \mathbf{z} \\
= & \int_{\mathbf{u}} \prod_{j}\left(\int_{z_{j}}\left(1+z_{j}\right)^{\frac{-1}{2}} \exp \left(\frac{-y_{j}^{2}}{2+2 z_{j}}\right) \mathrm{Ga}\left(z_{j} ; a, \max _{g: j \in g} u_{g}\right) \mathrm{d} z_{j}\right) \prod_{g} \mathrm{Ga}\left(u_{g} ; b, d\right) \mathrm{d} \mathbf{u}
\end{aligned}
$$

The rest of the proof is analogous to [6, Theorem 3]. The detailed proof is provided in Appendix B of the longer version of the paper [37].

By contrast, MRF is unable to drive down the amount of shrinkage when the response $y_{i}$ is large. To see the reason (e.g. for variable 1), note we fix $y_{2}$ to 1 . Since MRF enforces smoothness between $z_{1}$ and $z_{2}$, the fixed value of $y_{2}$ (hence its associated belief of $z_{2}$ ) will prevent $z_{1}$ to follow the increment of $y_{1}$, disallowing $z_{1}$ to utilize the heavy tail of horseshoe prior. The amount of shrinkage gets larger when $\alpha$ increases.

To summarize, among the three priors only the set cover prior enjoys all the three desirable properties namely conditional dependency, significant shrinkage for small observation, and vanishing shrinkage for large observations.

\section{Experimental Results}

We next study the empirical performance of SCP, compared with GCP, and MRF priors [34]. Since the MRF prior therein is restricted to spike and slab, to simplify comparison 

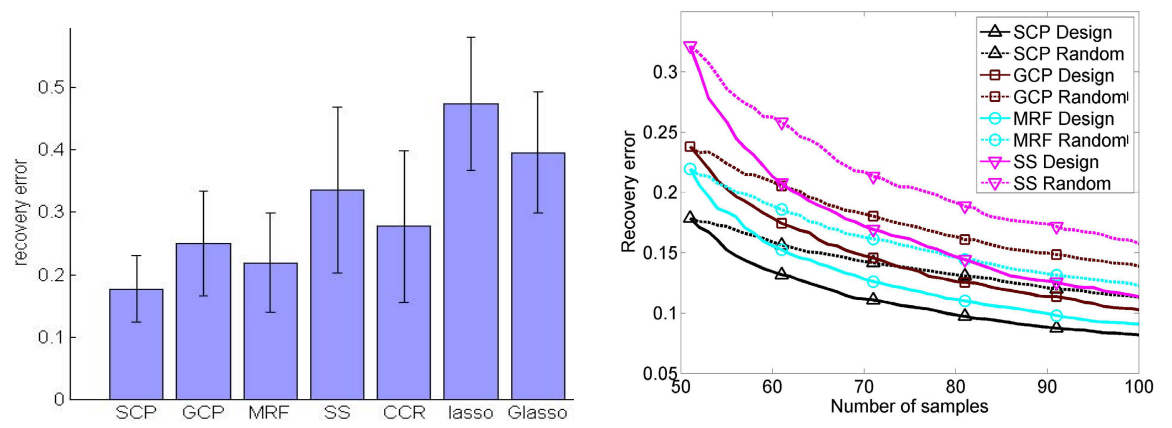

Fig. 10. Recovery rate for sparse signal

Fig. 11. Sequential experimental design for sparse recovery

we also base SCP and GCP on spike and slab. This allows convenient application of expectation propagation for posterior inference [EP, 39, 40], where all discrete factors are approximated by Bernoulli messages [34]. At each iteration, messages are passed from top to the bottom in Figure 4, and back up. Other inference algorithms are also possible, such as MCMC [e.g., 38], and variational Bayes [41]. Since the Bayesian models used here are typically multi-modal and the mean of the posterior is generally more important, we choose to use EP in our experiment, although it will also be interesting to try other methods.

Empirically EP always converged within 10 iterations, with change of message fallen below 1e-4. The loops make it hard to analyze the local or global optimality of EP result. But in practice, we did observe that with different initializations, EP always converged to the same result on all experiments, being highly reliable. To give an example of computational efficiency, in image denoising (Section 5.5, $p=n=4096$ ), it took only 0.5 seconds per image and per iteration to compute messages related to the prior, while common techniques for Gaussian likelihood allowed its related messages to be computed in 1-2 seconds.

As a baseline, we also tried spike and slab prior with non-overlapping groups (GSS) if reasonable non-overlapping group approximation is available, or even without groups (SS). Furthermore we consider three state-of-the-art frequentist methods, including Lasso, group Lasso (GLasso), and coding complexity regularization [CCR, 26]. Groups are assumed available as prior knowledge.

\subsection{Sparse Signal Recovery}

We first consider a synthetic dataset for sparse signal reconstruction with $p=82$ variables [42]. $\left\{\beta_{i}\right\}$ was covered by 10 groups of 10 variables, with an overlap of two variables between two successive groups: $\{1, \ldots, 10\},\{9, \ldots, 18\}, \ldots,\{73, \ldots, 82\}$. The support of $\boldsymbol{\beta}$ was chosen to be the union of group 4 and 5 , with the non-zero entries generated from i.i.d. Gaussian $\mathcal{N}(0,1)$. We used $n=50$ samples, with the elements of the design matrix $X \in \mathbb{R}^{n \times p}$ and the noisy measurements $\mathbf{y}$ drawn by

$$
X_{i j} \stackrel{i . i . d .}{\sim} \mathcal{N}(0,1), \quad \mathbf{y}=X \boldsymbol{\beta}+\boldsymbol{\epsilon}, \quad \epsilon_{i} \stackrel{i \text { i.i.d. }}{\sim} \mathcal{N}(0,1) .
$$


Table 1. Recovery error for network sparsity. GSS is not included as disjoint group approximation is not clear for general graph structure. CCR is also not included since its implementation in [26] does not allow flexible specification of groups.

\begin{tabular}{c|cccccc} 
& SCP & GCP & MRF & SS & Lasso & GLasso \\
\hline Jazz & $\mathbf{0 . 2 6 4} \pm 0.083$ & $0.312 \pm 0.068$ & $0.338 \pm 0.149$ & $0.398 \pm 0.188$ & $0.489 \pm 0.101$ & $0.456 \pm 0.107$ \\
NetScience & $\mathbf{0 . 0 6 7} \pm 0.005$ & $0.093 \pm 0.058$ & $0.167 \pm 0.110$ & $0.188 \pm 0.113$ & $0.394 \pm 0.045$ & $0.383 \pm 0.048$ \\
Email & $\mathbf{0 . 1 0 6} \pm 0.025$ & $\mathbf{0 . 1 0 4} \pm 0.054$ & $0.243 \pm 0.105$ & $0.310 \pm 0.130$ & $0.432 \pm 0.049$ & $0.420 \pm 0.057$ \\
C.elegans & $\mathbf{0 . 1 5 8} \pm 0.034$ & $0.163 \pm 0.025$ & $0.184 \pm 0.057$ & $0.225 \pm 0.101$ & $0.408 \pm 0.068$ & $0.394 \pm 0.068$
\end{tabular}

We used recovery error as the performance measure, which is defined as $\| \hat{\boldsymbol{\beta}}-$ $\boldsymbol{\beta}\left\|_{2} /\right\| \boldsymbol{\beta} \|_{2}$ for the posterior mean $\hat{\boldsymbol{\beta}}$. $X$ and $\boldsymbol{\beta}$ were randomly generated for 100 times, and we report the mean and standard deviation of recovery error. An extra 10 runs were taken to allow all models to select the hyper-parameters that optimize the performance on the 10 runs. This scheme is also used in subsequent experiments.

In Figure 10, SCP clearly achieves significantly lower recovery error than all other methods. MRF is the second best, followed by GCP. This suggests that when $\boldsymbol{\beta}$ is generated over the union of some groups, SCP is indeed most effective in harnessing this knowledge. Bayesian models for structured sparse estimation also outperform vanilla Bayesian models for independent variables (SS), as well as frequentist methods (CCR, Lasso, GLasso),

\subsection{Sequential Experimental Design}

A key advantage of Bayesian model is the availability of uncertainty estimation which facilitates efficient sequential experimental design [13]. We randomly generated a data pool of 10,000 examples based on (18), and initialized the training set with $n=50$ randomly selected examples (i.e. revealing their response $y_{i}$ ). Then we gradually increased the size of training set up to $n=100$. At each iteration, one example was selected and its response $y_{i}$ was revealed for training. In the random setting examples were selected uniformly at random, while in sequential experimental design, typically the example with the highest uncertainty was selected. For each candidate example $\mathbf{x}$, we used $\mathbf{x}^{\prime} V \mathbf{x}$ as the uncertainty measure, where $V$ is the current approximated posterior covariance matrix. The whole experiment was again repeated for 100 times, and the average recovery error is shown.

In Figure 11, for all models sequential experimental design is significantly more efficient in reducing the recovery error compared with random design. In particular, $\mathrm{SCP}$ achieves the steepest descent in error with respect to the number of measurements. This again confirms the superiority of SCP in modeling group structured sparsity in comparison to GCP and MRF. SS performs worst as it completely ignores the structure.

\subsection{Network Sparsity}

Following [34] and [43], we next investigate the network sparsity where each node is a variable and each edge constitutes a group (i.e. all groups have size 2). We tried on four network structures: Email ( $p=1,133$, \#edge=5,451), C.elegans (453, 2,015), 


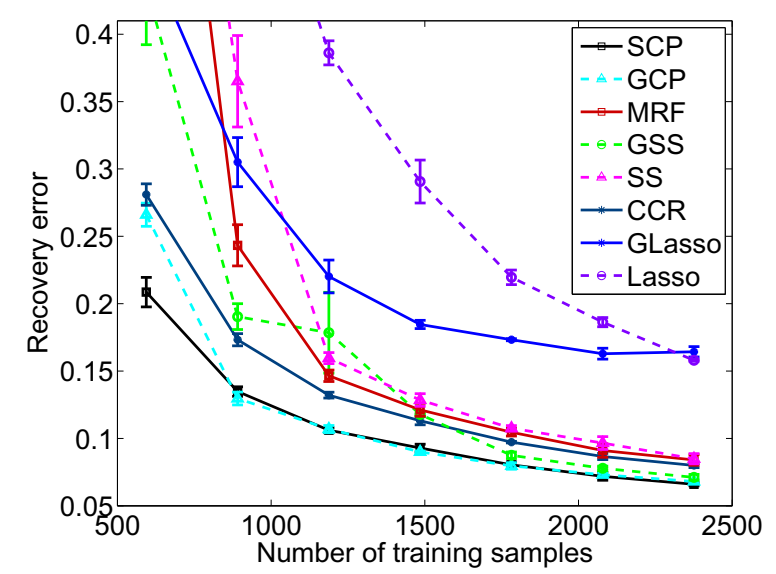

Fig. 12. Recovery error for background subtraction

Jazz $(198,2,742)$, NetScience $(1,589,2,742) .{ }^{3}$ See network properties in Table 1. We picked a subset of edges uniformly at random, and added their two incident nodes to the support of $\boldsymbol{\beta}$. By adjusting the number of selected edges, the size of the support of $\boldsymbol{\beta}$ is $0.25 p$, and the nonzero elements in $\boldsymbol{\beta}$ were sampled from $\mathcal{N}(0,1)$. The design matrix $X$ and the response $\mathbf{y}$ were drawn from (18). We used $n=\lfloor p / 2\rfloor$ examples as in [43].

The average recovery error of 100 runs is shown in Table 1. Again SCP yields significantly lower error than all other algorithms, except for a tie with GCP on Email. GCP outperforms MRF, which in turn defeats all other methods that do not faithfully model the group structure.

\subsection{Background Subtraction}

We next consider real-world applications in compressive sensing with overlapped group sparsity. Here the data generating process is beyond our control. In video surveillance, the typical configuration of the images are the sparse foreground objects on the static backgrounds. Our task here is to recover the sparse background subtracted images via compressive sensing.

Our experimental setting follows [26] $]^{4}$. The spatial consistency is an important prior knowledge on 2D image signals which has been successfully leveraged in various applications. Specifically pixels in a spatial neighborhood are likely to be background or foreground at the same time. Edges connecting pixels to its four neighbors are used in the MRF prior to encourage the consistency between adjacent pixels. For GSS which requires no overlap between groups, we simply defined the groups as non-overlapped $3 \times 3$ patches. For the rest structured priors, we defined groups as the overlapped $3 \times 3$ patches. Singleton groups were also added to deal with isolated foreground pixels. Each image is sized $80 \times 80(p=6,400)$. We varied the number of image $(n)$ from 600 to 2400.

\footnotetext{
${ }^{3}$ Downloaded from http: //www-personal . umich. edu/ mejn/netdata

${ }^{4}$ Video from http: //homepages.inf.ed.ac.uk/rbf/CAVIARDATA1
} 
Table 2. PSNR in image denoising. MRF and GSS are not included because in the case of hierarchical structure, it is not clear how to enforce MRF, or approximate a tree by disjoint groups.

\begin{tabular}{l|cccccc} 
& SCP & GCP & SS & Lasso & GLasso & CCR \\
\hline House & $\mathbf{2 8 . 7 7} \pm 0.04$ & $28.13 \pm 0.06$ & $27.72 \pm 0.06$ & $27.22 \pm 0.02$ & $27.24 \pm 0.03$ & $27.79 \pm 0.04$ \\
Lenna & $\mathbf{2 8 . 2 7} \pm 0.03$ & $27.65 \pm 0.02$ & $27.28 \pm 0.02$ & $26.95 \pm 0.03$ & $27.15 \pm 0.01$ & $27.11 \pm 0.02$ \\
pepperr & $\mathbf{2 6 . 5 7} \pm 0.03$ & $25.87 \pm 0.01$ & $25.75 \pm 0.03$ & $25.06 \pm 0.05$ & $25.39 \pm 0.06$ & $25.51 \pm 0.04$ \\
Boat & $\mathbf{2 6 . 8 0} \pm 0.01$ & $26.24 \pm 0.01$ & $26.09 \pm 0.01$ & $25.65 \pm 0.01$ & $26.05 \pm 0.02$ & $25.65 \pm 0.01$ \\
Barbara & $\mathbf{2 4 . 9 3} \pm 0.02$ & $24.56 \pm 0.02$ & $24.43 \pm 0.02$ & $24.23 \pm 0.01$ & $24.77 \pm 0.02$ & $24.34 \pm 0.01$
\end{tabular}

Figure 12 shows SCP and GCP achieve significantly lower recovery error than other methods on any number of measurement. The prior of spatial consistency does help improve the recovery accuracy, especially when the size of the training set is small. With sufficient training samples, both structured and non-structured methods can have accurate recovery. This can be seen by comparing Lasso with GLasso, as well as SCP with GCP, GSS, and SS. The superiority of SCP and GCP over GSS corroborates the importance of accommodating more flexible group definitions.

\subsection{Image Denoising with Tree-Structured Wavelets}

Our last set of experiment examines the effectiveness of structured sparse priors for modeling hierarchical sparsity. The task is to restore 2D images which are contaminated by noise via compressive sensing on 2D wavelet basis. The setting is similar to [26] and [21]. 2D wavelet basis at different resolution levels is used as dictionary to get sparse representation of images. There is a natural hierarchical structure in the wavelet coefficients: a basis $\mathbf{b}$ can be defined as the parent of all such basis at finer resolution and whose support is covered by the support of $\mathbf{b}$. Such tree-structured dependency corresponds to the nature of multi-resolution wavelet analysis and have been proven empirically effective in sparse representation of signals.

We choose the orthogonal Haar wavelet basis and the classical quad-tree structure on the 2D wavelet coefficients. We use PSNR $:=\log _{10}\left(\frac{255^{2}}{\mathrm{MSE}}\right)$ to measure the quality of recovery. The benchmark set consists of five standard testing images: house, Lenna, boat, Barbara and pepper. We added Gaussian white noise $\mathcal{N}\left(0,25^{2}\right)$ to the original images. The PSNR of the resulting noisy image is around 20. The images were divided into non-overlapped patches sized $64 \times 64$. Each patch is recovered independently with six levels of 2D Haar wavelet basis. For each method, we selected the parameters with the highest PSNR.

The recovery result is shown in Table 2. SCP delivers the highest PSNR in denoising on all test images, demonstrating the power of hierarchical structure prior to improve the recovery accuracy. Figure 15 in Appendix C of [37] shows a visual comparison of the denoising results, and it can be observed that SCP outperforms other methods in removing noise and preserving details in the image.

\section{Conclusion and Discussion}

We proposed a framework of set cover prior for modeling structured sparsity with overlapped groups. Its behavior is analyzed and empirically it outperforms existing competent 
structured priors. For future work, it will be interesting to further model sparsity within each group $[18,44]$. Extension to other learning tasks is also useful, e.g. multi-task learning $[45,46]$.

Acknowledgements. NICTA is funded by the Australian Government as represented by the Department of Broadband, Communications and the Digital Economy and the Australian Research Council through the ICT Centre of Excellence program.

\section{References}

[1] Bühlmann, P., van de Geer, S.: Statistics for High-Dimensional Data. Springer (2011)

[2] Eldar, Y., Kutyniok, G. (eds.): Compressed Sensing: Theory and Applications, Cambridge (2012)

[3] George, E., McCulloch, R.: Variable selection via Gibbs sampling. Journal of the American Statistical Association 88, 881-889 (1993)

[4] Mitchell, T.J., Beauchamp, J.J.: Bayesian variable selection in linear regression. Journal of the American Statistical Association 83(404), 1023-1032 (1988)

[5] Armagan, A., Dunson, D., Clyde, M.: Generalized Beta mixtures of Gaussians. In: NIPS (2011)

[6] Carvalho, C., Polson, N., Scott, J.: The horseshoe estimator for sparse signals. Biometrika 97, 465-480 (2010)

[7] Carvalho, C., Polson, N., Scott, J.: Handling sparsity via the horseshoe. In: AI-STATS (2009)

[8] Tibshirani, R.: Regression shrinkage and selection via the lasso. Journal of the Royal Statistical Society, Series B 58, 267-288 (1996)

[9] Tipping, M.: Sparse Bayesian learning and the relevance vector machine. Journal of Machine Learning Research 1, 211-244 (2001)

[10] Park, T., Casella, G.: The Bayesian lasso. Journal of the American Statistical Association 103(482), 618-686 (2008)

[11] Griffin, J., Brown, P.: Bayesian adaptive lassos with non-convex penalization. Australian \& New Zealand Journal of Statistics 53(4), 423-442 (2011)

[12] Fan, J., Li, R.: Variable selection via nonconcave penalized likelihood and its oracle properties. Journal of the American Statistical Association 96, 1348-1360 (2001)

[13] Hernández-Lobato, D., Hernández-Lobato, J.M., Dupont, P.: Generalized spike and slab priors for Bayesian group feature selection using expectation propagation. Journal of Machine Learning Research 16, 1891-1945 (2013)

[14] Ji, S., Dunson, D., Carin, L.: Multitask compressive sensing. IEEE Trans. Signal Processing 57(1), 92-106 (2009)

[15] Raman, S., Fuchs, T., Wild, P., Dahl, E., Roth, V.: The Bayesian group-lasso for analyzing contingency tables. In: ICML (2009)

[16] Yuan, M., Lin, Y.: Model selection and estimation in regression with grouped variables. Journal of the Royal Statistical Society, Series B 68(1), 49-67 (2006)

[17] Huang, J., Zhang, T.: The benefit of group sparsity. Annals of Stat. 38, 1978-2004 (2010)

[18] Yen, T.-J., Yen, Y.-M.: Grouped variable selection via nested spike and slab priors. ArXiv 1106.5837 (2011)

[19] Suo, Y., Dao, M., Tran, T., Srinivas, U., Monga, V.: Hierarchical sparse modeling using spike and slab priors. In: ICASSP (2013) 
[20] Li, C., Li, H.: Network-constrained regularization and variable selection for analysis of genomic data. Biometrics 24(9), 1175-1182 (2008)

[21] Jenatton, R., Mairal, J., Obozinski, G., Bach, F.: Proximal methods for hierarchical sparse coding. Journal of Machine Learning Research 12, 2297-2334 (2011)

[22] Li, F., Zhang, N.: Bayesian variable selection in structured high-dimensional covariate spaces with applications in genomics. Journal of the American Statistical Association 105(491), 1201-1214 (2010)

[23] Pan, W., Xie, B., Shen, X.: Incorporating predictor network in penalized regression with application to microarray data. Biometrics 66(2), 474-484 (2010)

[24] Stingo, F., Vannucci, M.: Variable selection for discriminant analysis with Markov random field priors for the analysis of microarray data. Bioinformatics 27(4), 495-501 (2011)

[25] Zhao, P., Rocha, G., Yu, B.: Grouped and hierarchical model selection through composite absolute penalties. Annals of Stat. 37(6A), 3468-3497 (2009)

[26] Huang, J., Zhang, T., Metaxas, D.: Learning with structured sparsity. Journal of Machine Learning Research 12, 3371-3412 (2011)

[27] Griffin, J., Brown, P.: Hierarchical sparsity priors for regression models. Arxiv:1307.5231 (2013)

[28] Yuan, M., Joseph, V.R., Zou, H.: Structured variable selection and estimation. Annals of Applied Statistics 3, 1738-1757 (2009)

[29] Griffin, J., Brown, P.: Some priors for sparse regression modelling. Bayesian Analysis 8(3), 691-702 (2013)

[30] Palmer, J.A., Wipf, D.P., Kreutz-Delgado, K., Rao, B.D.: Variational EM algorithms for non-Gaussian latent variable models. In: NIPS (2005)

[31] Bernardo, J., Smith, A.: Bayesian Theory. Wiley (1994)

[32] Griffin, J., Brown, P.: Inference with normal-gamma prior distributions in regression problems. Bayesian Analysis 5(1), 171-188 (2010)

[33] Obozinski, G., Bach, F.: Convex relaxation for combinatorial penalties. Technical Report HAL 00694765 (2012)

[34] Hernández-Lobato, J.M., Hernández-Lobato, D., Suárez, A.: Network-based sparse Bayesian classification. Pattern Recognition 44(4), 886-900 (2011)

[35] Argyriou, A., Foygel, R., Srebro, N.: Sparse prediction with the k-support norm. In: NIPS (2012)

[36] He, L., Carin, L.: Exploiting structure in wavelet-based Bayesian compressive sensing. IEEE Trans. Signal Processing 57(9), 3488-3497 (2009)

[37] Liu, X., Zhang, X., Caetano, T.: Bayesian models for structured sparse estimation via set cover prior. Technical report (2014), http://users.cecs.anu.edu.au/ ×zhang/papers/LiuZhaCae14_long.pdf

[38] Stingo, F., Chen, Y., Tadesse, M., Vannucci, M.: Incorporating biological information into linear models: A Bayesian approach to the selection of pathways and genes. Annals of Applied Statistics 5(3), 1978-2002 (2011)

[39] Minka, T.: A Family of Algorithms for Approximate Bayesian Inference. PhD thesis, MIT (2001)

[40] Seeger, M.: Bayesian inference and optimal design for the sparse linear model. Journal of Machine Learning Research 9, 759-813 (2008)

[41] Carbonetto, P., Stephens, M.: Scalable variational inference for bayesian variable selection in regression, and its accuracy in genetic association studies. Bayesian Analysis 7(1), 73$108(2012)$

[42] Jacob, L., Obozinski, G., Vert, J.-P.: Group lasso with overlap and graph lasso. In: ICML (2009)

[43] Mairal, J., Yu, B.: Supervised feature selection in graphs with path coding penalties and network flows. Journal of Machine Learning Research 14, 2449-2485 (2013) 
[44] Rockova, V., Lesaffre, E.: Incorporating grouping information in Bayesian variable selection with applications in genomics. Bayesian Analysis 9(1), 221-258 (2014)

[45] Hernández-Lobato, D., Hernández-Lobato, J.M.: Learning feature selection dependencies in multi-task learning. In: NIPS (2013)

[46] Hernández-Lobato, D., Hernández-Lobato, J.M., Helle-putte, T., Dupont, P.: Expectation propagation for Bayesian multi-task feature selection. In: ECML (2010) 